Number of Screening Rounds and Postscreening Prostate Cancer Incidence : Results from the Finnish Section of the European Randomized Study of Screening for Prostate Cancer Study

\author{
Pakarainen, Tomi
}

2016

Pakarainen, T, Raitanen, J , Talala , K , Taari , K, Kujala , P , Tammela , T L \& Auvinen , A 2016 , ' Number of Screening Rounds and Postscreening Prostate Cancer Incidence :

Results from the Finnish Section of the European Randomized Study of Screening for Prostate Cancer Study ' , European Urology , vol. 70 , no. 3 , pp. 499-505 . https://doi.org/10.1016/j.eururo.2016.05.

http://hdl.handle.net/10138/223948

https://doi.org/10.1016/j.eururo.2016.05.009

publishedVersion

Downloaded from Helda, University of Helsinki institutional repository.

This is an electronic reprint of the original article.

This reprint may differ from the original in pagination and typographic detail.

Please cite the original version. 


\title{
Number of Screening Rounds and Postscreening Prostate Cancer Incidence: Results from the Finnish Section of the European Randomized Study of Screening for Prostate Cancer Study
}

\author{
Tomi Pakarainen $^{a, b, *}$, Jani Raitanen $^{c, d}$, Kirsi Talala $^{e}$, Kimmo Taari $^{f, g}$, Paula Kujala ${ }^{h}$, \\ Teuvo L. Tammela ${ }^{a, b}$, Anssi Auvinen ${ }^{c}$ \\ ${ }^{a}$ Department of Urology, Tampere University Hospital, Tampere, Finland; ${ }^{\mathrm{b}}$ University of Tampere, School of Medicine, Tampere, Finland; ${ }^{\mathrm{c}}$ University of \\ Tampere, School of Health Sciences, Tampere, Finland; ${ }^{\mathrm{d}}$ UKK Institute for Health Promotion, Tampere, Finland; ${ }^{\mathrm{e}}$ Finnish Cancer Registry, Helsinki, Finland; \\ ${ }^{\mathrm{f}}$ Department of Urology, Helsinki University Hospital, Helsinki, Finland; ${ }^{\mathrm{g}}$ University of Helsinki, Helsinki, Finland; ${ }^{\mathrm{h}}$ Department of Pathology, FimLab \\ Laboratories, Tampere University Hospital, Tampere, Finland
}

\section{Article info}

\section{Article history:}

Accepted May 6, 2016

\section{Associate Editor:}

Matthew Cooperberg

\section{Keywords:}

Cancer incidence

ERSPC

Prostate cancer

PSA

Screening

\begin{abstract}
Background: The multicenter European Randomized Study of Screening for Prostate Cancer has shown a $21 \%$ reduction in prostate cancer (PC) mortality by prostate-specific antigen-based screening, with substantial overdiagnosis. In the present study, we analyzed the incidence of PC after screening in relation to the number of screening rounds attended in the Finnish section of the trial.

Objective: To evaluate the possible reduction in PC incidence following completed screening cycles in relation to the number of screening rounds attended.

Design, setting, and participants: The participants in the screening arm of the Finnish screening trial (29 298 men) were divided into subgroups of men who had participated at one, two, or three screening rounds. A reference group was formed of the 43151 men in the control arm by selecting age-matched controls for each subgroup of the screening participants. PC cases diagnosed after screening were identified from the Finnish Cancer Registry until the end of 2011. Follow-up of the screened men started 12 mo (365 d) after the last screening attendance and a similar date was assigned to the men in the control arm. Results and limitations: A total of 1514 new PCs cases (cumulative incidence 5.2\%) were diagnosed among the screened men after the last screening attendance. In the reference group formed from the control arm, 2683 cases (6.2\%) occurred. The hazard ratio (HR) for PC among nonparticipants in the screening arm was 0.89 (95\% confidence interval [CI] 0.79-0.99) compared with their controls. Among participants, the HR in those who participated once was 1.39 (95\% CI 1.22-1.57), among men who participated twice the HR was 0.97 (95\% CI 0.86-1.10), and among men screened three times the HR was 0.57 (95\% CI 0.49-0.68). A limitation of the study was that the comparison by attendance is not based on randomization.

Conclusions: The postscreening PC incidence is reduced after attending three screening rounds, but not after only one or two rounds. Thus, the increased cancer detection at screening is compensated by a subsequent risk reduction only after repeated screening cycles.

Patient summary: The results of the study indicate that at least three prostate-specific antigen-based screening cycles are needed to reduce subsequent prostate cancer incidence.

(C) 2016 European Association of Urology. Published by Elsevier B.V. All rights reserved.

* Corresponding author. Tampere University Hospital, Deptartment of Urology, Teiskontie 35, Tampere 33520, Finland. Tel. +358-3-311-65017; fax: +358-3-311-64256.

E-mail address: tomi.pakarainen@pshp.fi (T. Pakarainen).
\end{abstract}




\section{Introduction}

Prostate cancer (PC) is the most common cancer among men in most industrialized countries [1]. The general availability of prostate-specific antigen (PSA) testing in the early 1990s revolutionized the detection of PC. PSA testing increased the number of new cancers detected, particularly local disease [2]. Subsequently, a debate on the pros and cons of PSA screening has been ongoing, addressing mortality reduction versus overdiagnosis and overtreatment, as well as the overall balance in terms of quality-oflife effects and cost-effectiveness.

The European Randomized Study of Screening for Prostate Cancer (ERSPC) has been carried out in eight European countries since the 1990s. The aim of the trial is to evaluate the effect of PSA-based screening on PC mortality. The results have indicated a $21 \%$ relative $P C$ mortality reduction in the screening arm at $13 \mathrm{yr}$ of follow-up [3], while no such reduction has been observed in the US Prostate, Lung, Colorectal, and Ovarian trial [4].

Overdiagnosis is a major adverse effect of PC screening and it refers to the detection of cases that would not have surfaced clinically during the man's lifetime in the absence of screening. Screening theory assumes that screening harvests early cases and is followed by a compensatory reduction in the subsequent disease incidence. Detection of indolent, nonprogressive cases would not affect postscreening disease risk.

In this study, we analyzed PC incidence after screening among men screened once, twice, or three times, relative to a matched reference group selected from the control arm within the Finnish section of the ERSPC.

\section{Patients and methods}

The Finnish section of the ERSPC is the largest component of the ERSPC study, including 80458 men. Annually from 1996 to 1999, 8000 men aged $55 \mathrm{yr}, 59 \mathrm{yr}, 63 \mathrm{yr}$, and $67 \mathrm{yr}$ were randomly allocated into the screening arm, while the remainder of the birth cohorts formed the control arm. Men with a previous PC diagnosis were excluded. The men were reinvited for screening $4 \mathrm{yr}$ and $8 \mathrm{yr}$ after the first round, except for men aged $67 \mathrm{yr}$ at entry who were reinvited only once. The study protocol has been described in more detail previously [5,6].

In the present study, the men in the screening arm were divided into subgroups according to the number of screening rounds attended (screened once, twice, three times, or not at all). For each subgroup, an age-matched reference group from the control arm was randomly selected (Fig. 1).

The data on PC incidence were acquired from the Finnish Cancer Registry, a nationwide population-based registry with $98 \%$ completeness of all solid cancers in Finland. Follow-up for cancer incidence started at $12 \mathrm{mo}$ after the screening date ( $365 \mathrm{~d}$ after the last PSA measurement) to exclude screen-detected cancers. Hence, men with screen-detected

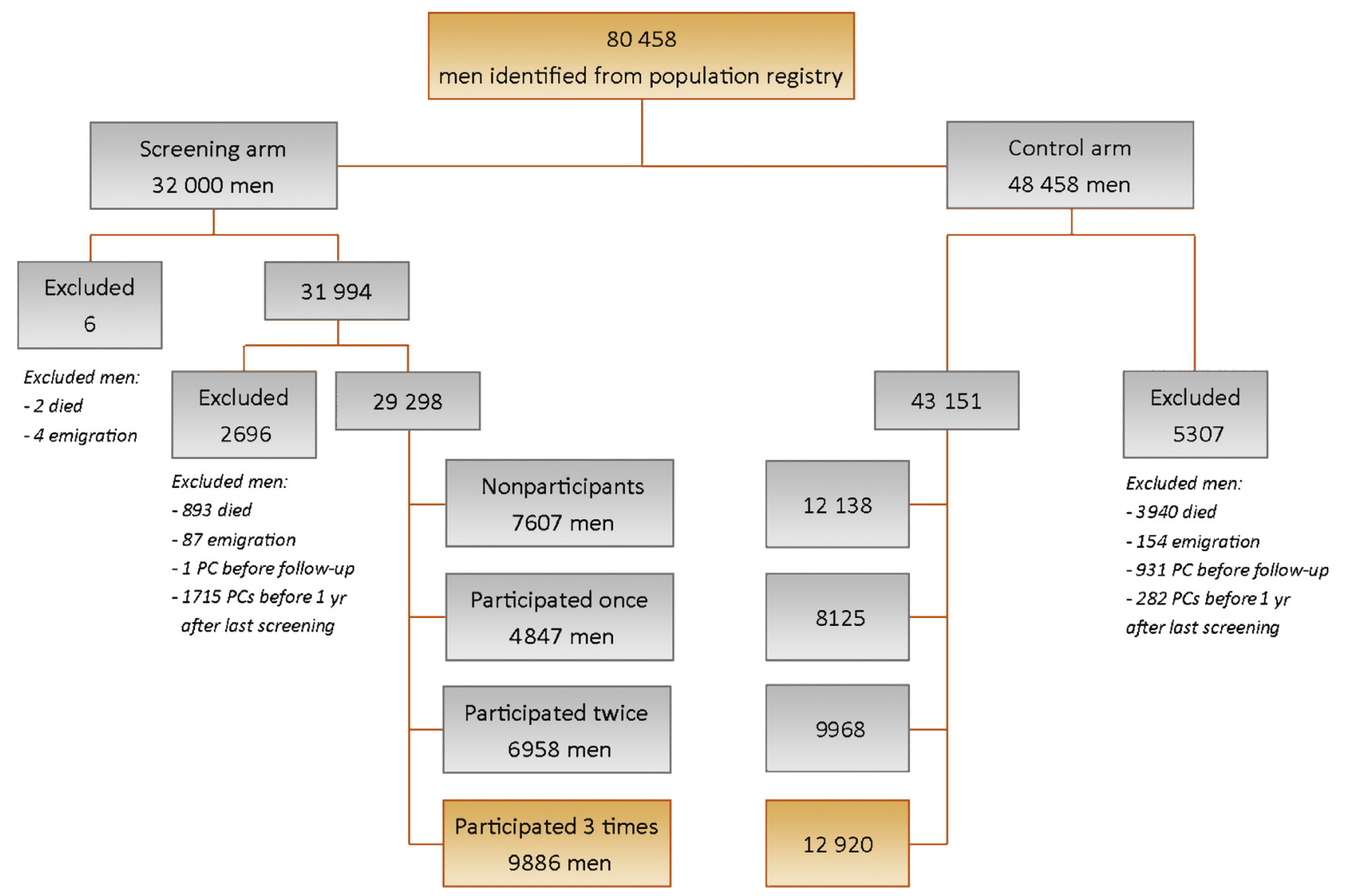

Fig. 1 - Flow chart of the trial population included in the analysis. For each group in the screening arm with a known number of screening attendances, a corresponding group of men was randomly selected as a reference from the control arm. Exclusion criteria were prostate cancer (PC) diagnosis before randomization, emigration, death, or PC diagnosis during $1 \mathrm{yr}$ after the last screening round. 
cancers $(N=1716)$ were not included in the analysis. In addition, men who emigrated or died before the start of follow-up were excluded. For the control group, a start date was assigned randomly from the start dates in the corresponding subgroup of the men in the screening arm. Additional analyses were also carried out for high-grade (Gleason $4+3$ or higher) cancers only, as well as PC incidence in two subgroups by age at entry ( $55 \mathrm{yr}$ and $59 \mathrm{yr}$ vs $63 \mathrm{yr}$ and $67 \mathrm{yr}$ at baseline). In addition, PC incidences at median follow-up time were analyzed. We also compared men invited twice and three times (regardless of attendance) relative to similar men in the control arm. As an ancillary analysis, we also repeated the analysis with adjustment for age.

PC incidence was analyzed using Cox regression, with hazard ratios (HR) and their 95\% confidence intervals (CI) estimated using Stata 12.0 (StataCorp, College Station, TX, USA).

\section{Results}

A total of 29298 men in the screening arm and 43151 men in the control arm were included (Fig. 1), with 1514 PC cases in the screening arm diagnosed at least 12 mo after their last screening (last PSA measurement date; cumulative incidence 5.2\%) versus 2683 cases in the control arm (6.2\%; HR $0.94,95 \% \mathrm{CI} 0.88-1.00)$. The median length of follow-up ranged from $11.8 \mathrm{yr}$ among never screened to $4.7 \mathrm{yr}$ in those screened three times (with a similar duration of follow-up in each control group; Table 1 ).

Postscreening PC incidence was increased only among men screened once compared with their controls. The incidence rate for PC among the men invited for screening who never participated was 6.3 cases/1000 person-yr versus 7.1 per 1000 in their control group with a similar age distribution and start of follow-up (Table 2). Correspondingly, the risk for PC in men who were screened once was 11.2 versus 8.1 cases/1000 person-yr among their controls, for men screened twice 8.9 versus 9.1 cases/1000 person-yr and those who participated three times 4.5 versus 7.9 cases/1000 person-yr (Fig. 2, Table 3). PC incidence rates at median follow-up time were in line with postscreening incidence rates (Table 1 ).

The men who did not participate had a similar PC risk as their controls, regardless of age at entry: PC incidence 8.6 versus 9.8 cases/1000 person-yr in men aged $63 \mathrm{yr}$ or $67 \mathrm{yr}$ and 4.9 versus 5.4 cases per 1000 in those aged $55 \mathrm{yr}$ or $59 \mathrm{yr}$. Also, the excess incidence among the men screened once was comparable for men aged 63-67 yr and 55-59 yr (14.9 vs $9.8 \mathrm{yr}$, and $8.5 \mathrm{vs} 6.7 \mathrm{yr}$, respectively). In the men screened twice, there was a reduction in the older age groups ( 7.9 vs $11.7 \mathrm{yr}$ ), but excess incidence in the younger men. For the men screened three times, a reduction in the subsequent PC incidence was observed in both age groups (4.9 vs $10.5 \mathrm{yr}$ in older men and $4.4 \mathrm{vs} 7.1 \mathrm{yr}$ in younger men; Fig. 3, Table 3 ).

In the analysis of high-grade cancers, a reduction of PC incidence was seen only in men screened twice or three times ( 2.3 vs 3.2 cases/1000 person-yr, and 1.5 vs 2.7 cases/ 1000 person-yr, respectively). In contrast, no effect on incidence of high-grade PC was found in men who refused screening ( 2.1 vs 2.2 cases/1000 person-yr) or participated only once (2.3 vs 2.3 cases/1000 person-yr; Fig. 4).

In the analysis by number of screening invitations, regardless of screening attendance, the men invited twice did not show a significant difference in PC incidence compared with their controls (HR 1.11, 95\% CI 0.99-1.25), while men invited three times had a reduced incidence (HR 0.66, 95\% CI 0.58-0.74). In an additional analysis with adjustment for age instead of using age-matched controls for each screened man, the results were practically identical to the main analysis (data not shown).

Table 1 - Number of participants in different subgroups ( $n$ ), median follow-up times for men without prostate cancer (PC) diagnosis (yr), incidence rates for PC at median follow-up time (cases/1000 men/yr), over-all mortality (number of men and mortality percentage [\%]) of the subgroups, number of PCs, and number of high-grade PCs

\begin{tabular}{|c|c|c|c|c|c|c|c|}
\hline & & $n$ & Median follow-up time & Incidence rate & Over-all mortality (\%) & PC & High-grade PC \\
\hline \multirow[t]{2}{*}{ Nonparticipants } & Screening arm & 7607 & 11.8 & $6.3(p=0.14)$ & 2945 (39) & 469 & 161 \\
\hline & Control arm & 12138 & 12.3 & 7.1 & $2934(24)$ & 955 & 299 \\
\hline \multirow[t]{2}{*}{ Participated once } & Screening arm & 4847 & 8.3 & $12.0(p<0.001)$ & $1715(35)$ & 419 & 105 \\
\hline & Control arm & 8125 & 11.2 & 8.2 & $1836(23)$ & 629 & 186 \\
\hline \multirow[t]{2}{*}{ Participated twice } & Screening arm & 6958 & 7.6 & $9.1(p=0.7)$ & $1577(23)$ & 412 & 117 \\
\hline & Control arm & 9968 & 7.7 & 9.3 & $2023(20)$ & 629 & 235 \\
\hline \multirow[t]{2}{*}{ Participated three times } & Screening arm & 9886 & 4.8 & $4.5(p<0.001)$ & $703(7)$ & 214 & 71 \\
\hline & Control arm & 12920 & 4.7 & 8.0 & $1322(10)$ & 470 & 168 \\
\hline
\end{tabular}

Table 2 - Prostate cancer (PC) incidence by trial arm and number of screening rounds attended after the last screening attendance (or corresponding date in the control arm) until the end of the year 2011

\begin{tabular}{|c|c|c|c|c|c|c|c|}
\hline & \multicolumn{3}{|c|}{ Screening arm } & \multicolumn{3}{|c|}{ Control arm } & \\
\hline & $\begin{array}{c}\text { Number of } \\
\text { men }\end{array}$ & $\begin{array}{l}\text { Number of } \\
\text { PC cases }\end{array}$ & $\begin{array}{l}\text { Cumulative } \\
\text { incidence (\%) }\end{array}$ & $\begin{array}{c}\text { Number of } \\
\text { men }\end{array}$ & $\begin{array}{c}\text { Number of } \\
\text { PC cases }\end{array}$ & $\begin{array}{l}\text { Cumulative } \\
\text { incidence (\%) }\end{array}$ & \\
\hline Nonparticipants & 7607 & 469 & 6.2 & 12138 & 955 & 7.9 & $p=0.03$ \\
\hline Participated once & 4847 & 419 & 8.6 & 8125 & 629 & 7.7 & $p<0.001$ \\
\hline Participated twice & 6958 & 412 & 5.9 & 9968 & 629 & 6.3 & $p=0.6$ \\
\hline Participated three times & 9886 & 214 & 2.2 & 12920 & 470 & 3.6 & $p<0.001$ \\
\hline
\end{tabular}



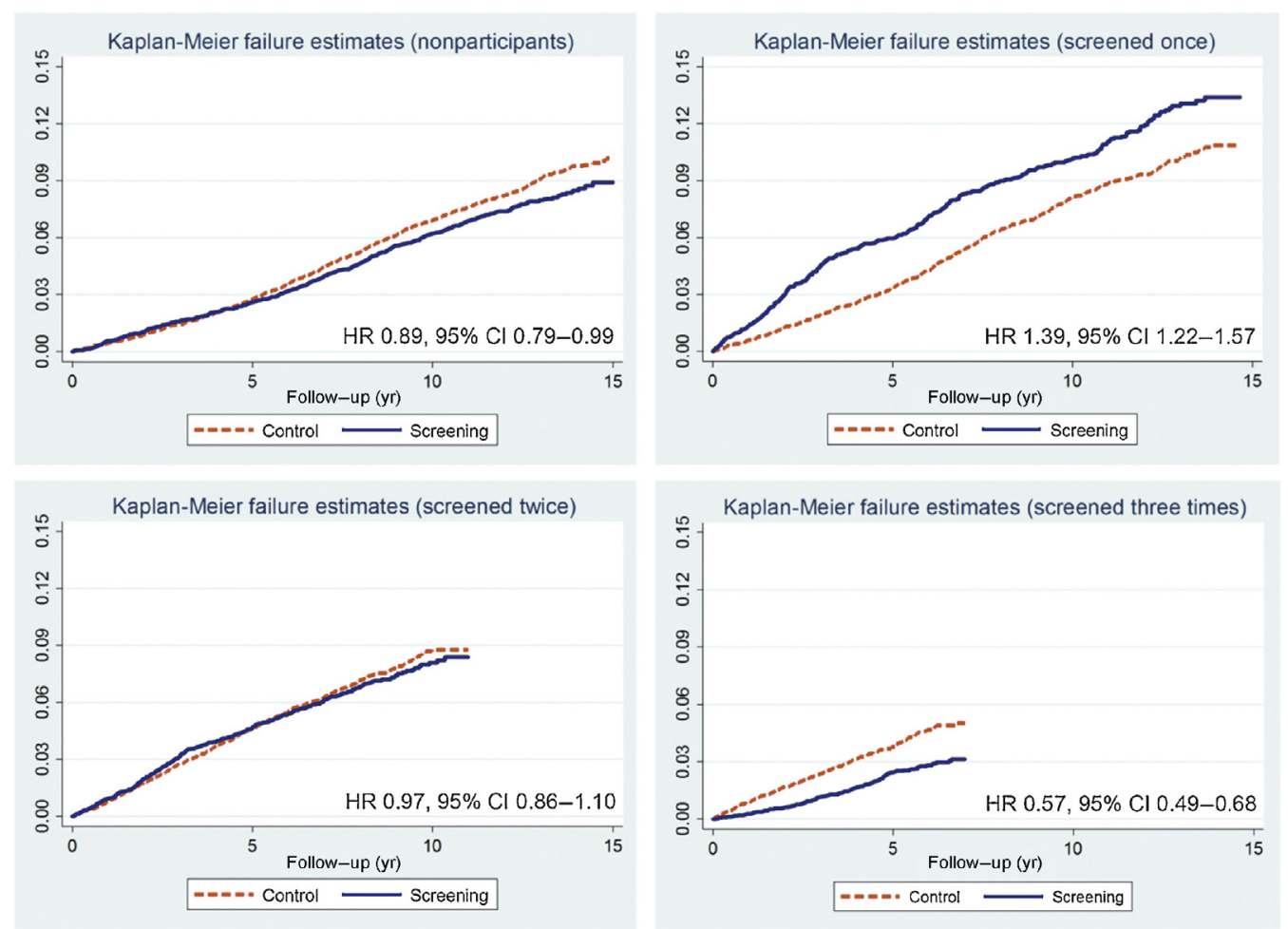

Fig. 2 - Cumulative incidence of prostate cancer after last screening by number of screening attendances in the screening arm and corresponding men in the control arm.

$\mathrm{CI}=$ confidence interval; $\mathrm{HR}=$ hazard ratio.

Table 3 - Prostate cancer incidence rate by trial arm and number of screening rounds attended after the last screening attendance (or corresponding date in the control arm) until the end of 2011, with hazard ratio (HR) and 95\% confidence interval (CI). The analysis has been done with all men together and additional subanalysis with men divided in younger and older age groups (born 1929-1936 and 1937-1944)

\begin{tabular}{|c|c|c|c|c|c|}
\hline & & Screening arm (cases/1000 men/yr) & Control arm (cases/1000 men/yr) & HR & $95 \% \mathrm{CI}$ \\
\hline \multirow{3}{*}{ Nonparticipants } & All & 6.3 & 7.1 & 0.89 & $0.79-0.99$ \\
\hline & 1929-1936 & 8.6 & 9.8 & 0.88 & $0.75-1.02$ \\
\hline & 1937-1944 & 4.9 & 5.4 & 0.92 & $0.79-1.08$ \\
\hline \multirow[t]{3}{*}{ Participated once } & All & 11.2 & 8.1 & 1.39 & $1.22-1.57$ \\
\hline & 1929-1936 & 14.9 & 9.8 & 1.50 & $1.27-1.78$ \\
\hline & 1937-1944 & 8.5 & 6.7 & 1.29 & $1.07-1.55$ \\
\hline \multirow[t]{3}{*}{ Participated twice } & All & 8.9 & 9.1 & 0.97 & $0.86-1.10$ \\
\hline & 1929-1936 & 7.9 & 11.7 & 0.67 & $0.57-0.79$ \\
\hline & $1937-1944$ & 10.4 & 6.1 & 1.69 & $1.38-2.06$ \\
\hline \multirow[t]{3}{*}{ Participated three times } & All & 4.5 & 7.9 & 0.57 & $0.49-0.68$ \\
\hline & 1929-1936 & 4.9 & 10.5 & 0.46 & $0.34-0.63$ \\
\hline & $1937-1944$ & 4.4 & 7.1 & 0.62 & $0.51-0.75$ \\
\hline
\end{tabular}

\section{Discussion}

A successful screening program should be able to detect cases in the preclinical phase before they are detected clinically, and hence reduce the subsequent incidence of the disease. Our present findings indicate that a single screening round is not sufficient for detecting a sufficiently large fraction of the pool of preclinical disease to achieve such an effect. Our results show a decreased PC incidence after three screening rounds, while the incidence was increased following a single screening round. These findings suggest that a compensatory reduction can be achieved only after repeated screening, consistent with the notion that a single (prevalence) screen harvests more from the prevalence pool of indolent cases, and only repeated screening is able to detect a substantial proportion of progressive cancers, probably de novo cases arising between the screening rounds.

Our results also showed a higher cumulative PC incidence after screening following one to two screening rounds than the full three rounds, but this is due to a shorter follow-up. In addition, the age distribution differed, as the oldest men were invited only twice (up to age $71 \mathrm{yr}$ ). However, the comparison to the control group was not affected by these differences, as the control groups were formed within the control arm for each subgroup of the 
Yr of birth 1929-1936

1937-1944

Nonparticipants
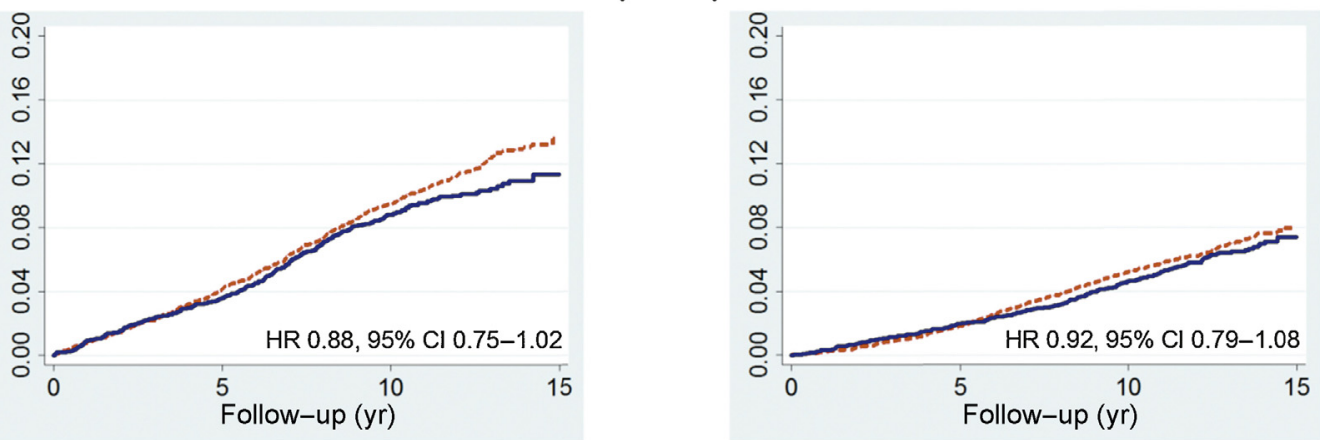

\section{Screened once}
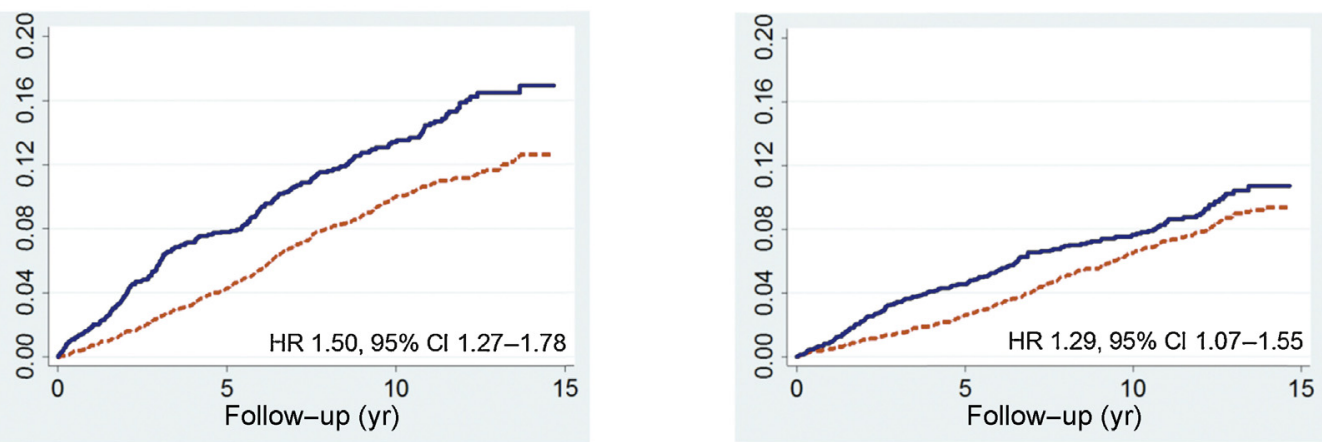

\section{Screened twice}
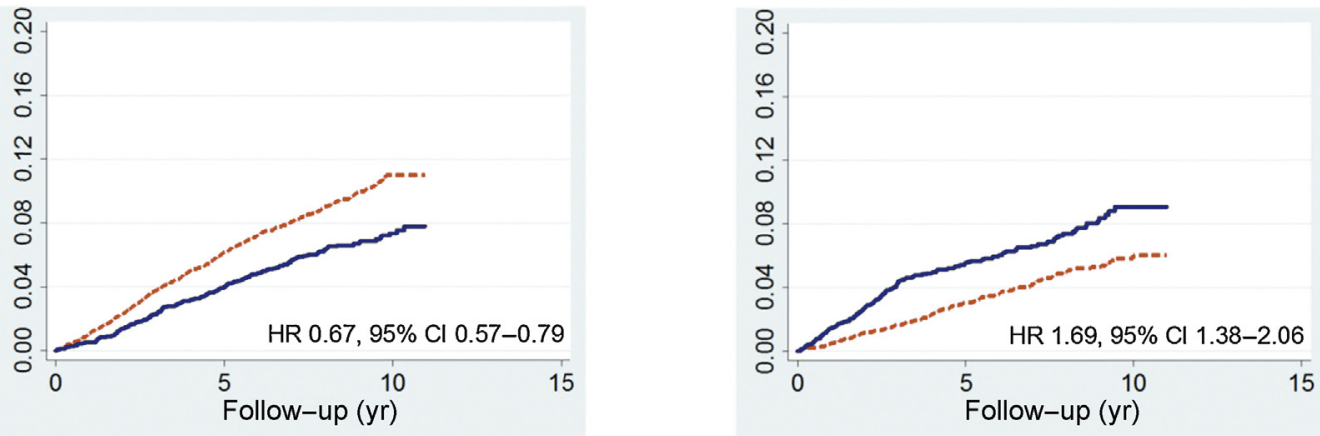

Screened three times
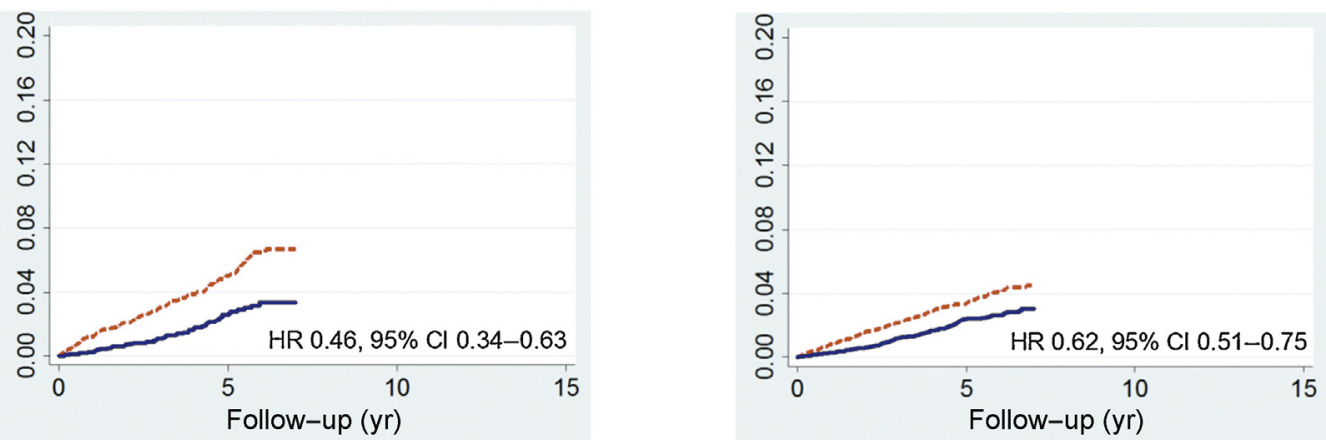

Fig. 3 - Nelson-Aalen estimates of cumulative prostate cancer incidence by year of birth, trial arm, and number of screening rounds attended after the last screening round (or corresponding date in the control arm). Continuous blue line indicates screened men. Orange broken line indicates control men.

$\mathrm{CI}=$ confidence interval; $\mathrm{HR}=$ hazard ratio. 

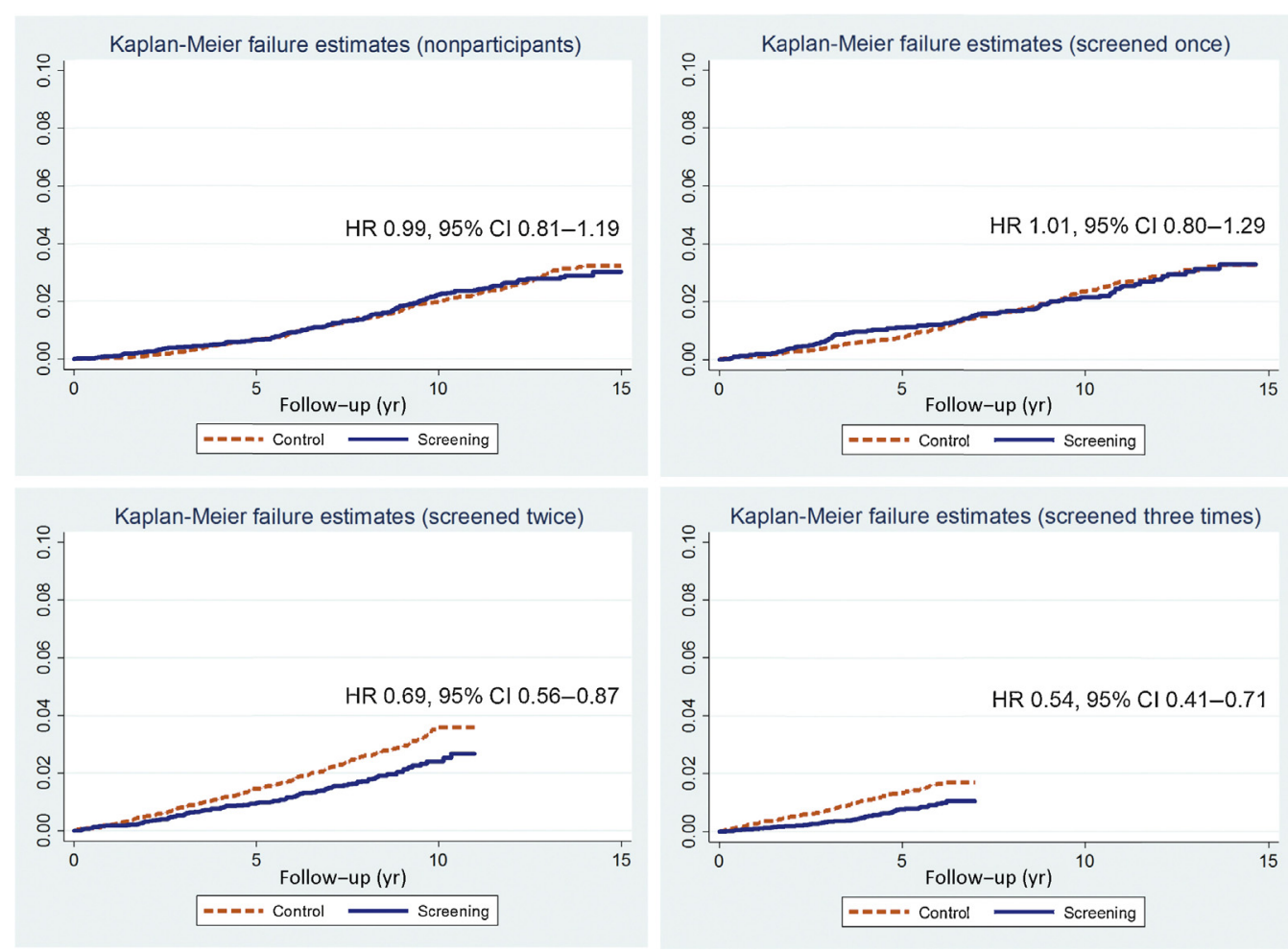

Fig. 4 - Cumulative incidence of high-grade prostate cancer after screening rounds. $\mathrm{CI}=$ confidence interval; $\mathrm{HR}=$ hazard ratio .

screened men to provide a similar age distribution and start of follow-up.

The screen-detected cases were not included in the analysis, but follow-up was started at 12 mo after the screening. Therefore, the findings (including a higher cumulative risk in the nonscreened men than any of the screened groups within the screening arm) do not mean that the reduced PC risk postscreening would be sufficient to counterbalance the overdiagnosis at screening.

Our findings differ slightly from the Swedish results [7] regarding PC incidence in relation to the number of screening rounds. Our findings indicated a reduction in PC risk after screening for the men who attended all three screening cycles. In contrast, the incidence was higher among the men who participated in screening only once. Thus, if the number of screening cycles is not taken into account, our results might be parallel to the results of Bergdahl et al. [7].

The men who were screened only once showed an increased PC incidence, which is probably attributable mainly to intensive follow-up after an elevated PSA or a negative biopsy.

After the second screening round, no difference was seen between the screened and control men. However, the subanalysis showed an increased risk for PC in younger men, while older men had diminished PC incidence. The reasons for this may be multifactorial. The difference between the subgroups could probably be partly due to a shorter follow-up time in the older men, and in addition higher PSA reference values in older men possibly raise the threshold for referring an older man for prostate biopsies. Furthermore, it is possible that $\mathrm{PC}$ in younger men is more aggressive and rapidly progressing and thus screening detects fewer cases in the younger ages.

The men who did not comply with the screening invitations are likely to include both those who already had a recent PSA test, but mainly men who have opted against PSA testing either for practicalities (inconvenient time) or lack of interest (small perceived PC risk and/or benefit of screening). In general, screening nonparticipation is more common in lower socioeconomic groups, and also in the Finnish trial clear differences in participation by level of income and education were shown [8]. The effect of socioeconomic status (SES) on the risk of PC varies by stage-a high SES is associated with a higher risk of early PC, but a decreased risk of advanced or high-risk disease [812]. Hence, men with lower SES are both less likely to attend screening and have a higher risk of advanced PC. This can distort the comparison of PC risk in screened and nonscreened men, exaggerating the overdiagnosis, and underestimating the reduction in advanced disease. Hence, adjustment for SES should ideally be used in comparisons of screened and nonscreened men.

Comparison of subgroups by screening attendance may be affected by selection bias due to lack of comparability. Therefore, an alternative analysis was carried out by comparing men invited twice and three times. Similarly to the main analysis, only in men invited three times had a significant decrease in PC incidence compared with their controls. This suggests that the differences between men by 
screening attendance do not account for the reduction in PC incidence after repeated screening.

The findings of our study are also relevant for the British PC trial [13], as the present results indicate that a single screening round may be insufficient to detect a substantial proportional of progressive preclinical PCs surfacing after screening.

Our findings demonstrate that the postscreening PC incidence is reduced in men who participated in all three screening rounds of the Finnish trial. The effect was not seen after one to two rounds. In addition, the incidence on high Gleason grade cancers was not diminished after a single screening round, but decreased after two to threerounds. Thus, these results indicate that at least three screening cycles are needed for subsequent reduction of overall PC incidence and a minimum of two rounds to decrease highgrade cancers. Whether the number of screening rounds influences PC mortality needs further evaluation.

Author contributions: Tomi Pakarainen had full access to all the data in the study and takes responsibility for the integrity of the data and the accuracy of the data analysis.

Study concept and design: Auvinen,Tammela,Pakarainen.

Acquisition of data: Tammela, Taari, Kujala, Talala.

Analysis and interpretation of data: Auvinen, Tammela, Pakarainen.

Drafting of the manuscript: Pakarainen, Auvinen.

Critical revision of the manuscript for important intellectual content: Pakarainen, Raitanen, Talala, Taari, Kujala, Tammela, Auvinen.

Statistical analysis: Raitanen.

Obtaining funding: Auvinen, Tammela, Taari.

Administrative, technical, or material support: None.

Supervision: Auvinen, Tammela.

Other: None.

Financial disclosures: Tomi Pakarainen certifies that all conflicts of interest, including specific financial interests and relationships and affiliations relevant to the subject matter or materials discussed in the manuscript (eg, employment/affiliation, grants or funding, consultancies, honoraria, stock ownership or options, expert testimony, royalties, or patents filed, received, or pending), are the following: None.

Funding/Support and role of the sponsor: The study was funded by the grant of Academy of Finland (Grant number 260931) and grants from the Cancer Society of Finland and Tampere University Hospital/Pirkanmaa Hospital District.

\section{References}

[1] Center MM, Jemal A, Lortet-Tieulent J, et al. International variation in prostate cancer incidence and mortality rates. Eur Urol 2012; 61:1079-92.

[2] Welch HG, Albertsen PC. Prostate cancer diagnosis and treatment after the introduction of prostate-specific antigen screening. J Natl Cancer Inst 2009;101:1325-9.

[3] Schröder FH, Hugosson J, Roobol MJ, et al. Screening and prostate cancer mortality: Results of the European Randomized Study of Screening for Prostate Cancer (ERSPC) at 13 years of follow-up. Lancet 2014;384:2027-35.

[4] Andriole GL, Crawford ED, Grubb RL, et al. Mortality results from a randomized prostate cancer screening trial. N Engl J Med 2009;360: 1310-9.

[5] Finne P, Stenman UH, Määttänen L, et al. The Finnish trial of prostate cancer screening: Where are we now? BJU Int 2003;92(Suppl 2): $22-6$.

[6] Kilpeläinen TP, Tammela TL, Malila N, et al. Prostate cancer mortality in the Finnish randomized screening trial. J Natl Cancer Inst 2013;105:719-25.

[7] Bergdahl AG, Holmberg E, Moss S, Hugosson J. Incidence of prostate cancer after termination of screening in population-based randomized screening trial. Eur Urol 2013;64:703-9.

[8] Kilpeläinen T, Talala K, Raitanen J, et al. Prostate cancer and socioeconomic status in the Finnish randomized study of screening for prostate cancer. Am J Epidemiol. In press.

[9] Steenland K, Rodriguez C, Mondul A, Calle EE, Thun M. Prostate cancer incidence and survival in relation to education (United States). Cancer Causes Control 2004;9:939-45.

[10] Cheng I, Witte JS, McClure LA, et al. Socioeconomic status and prostate cancer incidence and mortality rates among the diverse population of California. Cancer Causes Control 2009;8:1431-40.

[11] Clegg LX, Reichman ME, Miller BA, et al. Impact of socioeconomic status on cancer incidence and stage at diagnosis: selected findings from the surveillance, epidemiology, and end results: National Longitudinal Mortality Study. Cancer Causes Control 2009;4: 417-35.

[12] Rundle A, Neckerman KM, Sheehan D, et al. A prospective study of socioeconomic status, prostate cancer screening, and incidence among men at high risk for prostate cancer. Cancer Causes Control 2013;2:297-303.

[13] Turner EL, Metcalfe C, Donovan JL, et al. Design and preliminary recruitment results of the Cluster randomized triAL of PSA testing for Prostate Cancer (CAP). Br J Cancer 2014;110:2829-36. 